\title{
Tacrolimus increases the expression level of the chemokine receptor CXCR2 to promote renal fibrosis progression
}

\author{
DONGDONG WANG ${ }^{1}, \mathrm{XIAO} \mathrm{CHEN}^{1}, \mathrm{MENG} \mathrm{FU}^{1}, \mathrm{HONG} \mathrm{XU}^{2}$ and ZHIPING LI ${ }^{1}$ \\ Departments of ${ }^{1}$ Pharmacy and ${ }^{2}$ Nephrology, Children's Hospital of Fudan University, Shanghai 201102, P.R. China
}

Received July 4, 2019; Accepted September 24, 2019

DOI: $10.3892 / \mathrm{ijmm} .2019 .4368$

\begin{abstract}
Tacrolimus is one of the most used and effective immunosuppressive agents currently available in the clinic; however, its use is limited by nephrotoxicity, which is the main secondary effect of this drug. The mechanisms underlying tacrolimus-induced nephrotoxicity remain unknown. The present study aimed to investigate the mechanism underlying tacrolimus-induced nephrotoxicity and to identify novel potential targets. Masson staining, Sirius red staining and periodic acid-silver methenamine staining were used to observe kidney pathological changes. Immunohistochemical and immunofluorescent analyses were performed to examine the expression levels of vimentin, E-cadherin and $\alpha$-smooth muscle actin $(\alpha$-SMA). Transcriptomics and bioinformatics analyses were performed to investigate the nephrotoxicity mechanism induced by tacrolimus using RNA-sequencing, differentially expressed genes identification and annotation, and Kyoto Encyclopedia of Genes and Genomes pathway enrichment analysis. The present results demonstrated that compared with the normal control group, the tacrolimus nephrotoxicity group exhibited severe renal fibrosis $(\mathrm{P}<0.05)$, upregulated vimentin $(\mathrm{P}<0.01)$, downregulated $\mathrm{E}$-cadherin $(\mathrm{P}<0.05)$ and upregulated $\alpha$-SMA $(\mathrm{P}<0.01)$. Transcriptomics and bioinformatics analyses identified the pathway "cytokine-cytokine receptor interaction' as the most significantly enriched $(\mathrm{P}<0.05)$. Moreover, KEGG pathway enrichment analysis identified that tacrolimus increased the expression levels of chemokine (C-X-C) motif ligand (CXCL)1, CXCL2 and CXCL3 and the chemokine receptor C-X-C chemokine receptor type 2 (CXCR2). Collectively, the present study suggested that tacrolimus
\end{abstract}

Correspondence to: Professor Hong Xu, Department of Nephrology, Children's Hospital of Fudan University, 399 Wanyuan Road, Shanghai 201102, P.R. China

E-mail: hxu@shmu.edu.cn

Professor Zhiping Li, Department of Pharmacy, Children's Hospital of Fudan University, 399 Wanyuan Road, Shanghai 201102, P.R. China

E-mail: zplifudan@126.com

Key words: transcriptomics, analysis, tacrolimus, C-X-C chemokine receptor type 2 , renal fibrosis increases the level of chemokine receptor CXCR2 to promote renal fibrosis progression, which is one of the potential mechanisms underlying tacrolimus-induced nephrotoxicity.

\section{Introduction}

Tacrolimus, a potent immunosuppressive agent, was developed in the 1990s and can be isolated from the bacteria Streptomyces tsukubaensis. FK-506-binding protein 12 (FKBP-12) is an important immunophilin targeted by tacrolimus in T cells, and tacrolimus can form a complex with FKBP-12, thus repressing the phosphatase calcineurin, an enzyme necessary to activate the nuclear factor of T cells (NF-AT) (1-4). NF-AT serves a key role in the transcription of cytokine-encoding genes in $\mathrm{T}$ cells (5).

Tacrolimus has primarily been used for treating patients who have received allogeneic organ transplants or patients with autoimmune diseases (4,6-11). However, clinical management of tacrolimus therapy can be challenging due to its narrow treatment range and significant variabilities within and among individuals $(12,13)$, and these limitations can be caused by multiple factors that interfere with its metabolism. Therefore, in clinical settings, therapeutic drug monitoring is used to optimize the treatment regimen (14).

Tacrolimus has various side effects, and nephrotoxicity is the most common, occurring in $~ 50 \%$ of patients treated with tacrolimus (15). Renal fibrosis is commonly associated with nephrotoxicity (16-20). Fibrosis plays a crucial role in cadmium-induced nephrotoxicity (16), cyclosporine nephrotoxicity $(17,19)$, nephrotoxicity induced by oral sodium nitrite (18) and aristolochic acid nephrotoxicity (20). In addition, renal fibrosis is an important process underlying tacrolimus nephrotoxicity $(21,22)$. Therefore, it is important to identify the factors that lead to renal fibrosis following tacrolimus nephrotoxicity and control its development $(21,22)$. The present study aimed to investigate the mechanism underlying renal fibrosis induced by tacrolimus and to identify novel potential targets.

\section{Materials and methods}

Animal experiments. In total, 16 specific pathogen free male Wistar rats (age, 3 weeks; weight, $64 \pm 3 \mathrm{~g}$ ) were purchased from Shanghai SLAC Laboratory Animal Co., Ltd. The animals were housed in standard cages and maintained 
under standard conditions at a constant room temperature of $20-25^{\circ} \mathrm{C}$, a humidity of $40-70 \%$ and a $12 / 12 \mathrm{~h}$ light/dark cycle. All rats had free access to regular chow and water. The rats were randomly divided into a normal control (NC) group $(n=8)$ and a tacrolimus nephrotoxicity $(N E)$ group $(n=8)$. The NE group was intraperitoneally injected with tacrolimus (Astellas Ireland Co., Ltd.) at $2 \mathrm{mg} / \mathrm{kg}$ per day $(23,24)$. The NC group received daily intraperitoneal administrations of equal volumes of tacrolimus solvent, which consisted of polyoxyethylene hydrogenated castor oil and absolute ethyl alcohol, for 2 weeks. Animal protocols and procedures were approved by The Animal Care and Use Committee of Children's Hospital of Fudan University (Shanghai, China) and complied with the appropriate institutional regulations.

Sample collection. After 2 weeks of intervention, Wistar rats were anesthetized with $10 \%$ chloral hydrate $(300 \mathrm{mg} / \mathrm{kg})$ by intraperitoneal injection. Animals did not present obvious signs of peritonitis in the present study. Blood samples were collected from the abdominal aorta. The rats were euthanized by cervical dislocation under deep anesthesia with $10 \%$ chloral hydrate. The death of rats was verified by the heartbeat, breathing and neural reflex. After Wistar rats were sacrificed, the kidneys were removed and weighed. The kidney index was calculated using the following formula: Kidney index = (average kidney weight/body weight) (25). Paraformaldehyde (4\%) was used to fix the fresh kidneys, and liquid nitrogen was used to rapidly freeze the residual kidney tissues, which were stored at $-80^{\circ} \mathrm{C}$ until further analysis.

Observation of kidney histological. Kidney tissues were fixed in $4 \%$ paraformaldehyde at $4^{\circ} \mathrm{C}$ for $24 \mathrm{~h}$, embedded in paraffin and cut into $4-\mu \mathrm{m}$-thick sections (26). The sections were then stained by Masson staining, Sirius red staining and periodic acid-silver methenamine (PASM) staining (all from Wuhan Servicebio Technology Co., Ltd.). Masson staining was performed at room temperature $\left(25^{\circ} \mathrm{C}\right)$, with iron hematoxylin for $3 \mathrm{~min}$, Ponceau $\mathrm{S}$ solution for $5 \mathrm{~min}$, phosphomolybdic acid for $1 \mathrm{~min}$ and aniline blue for $3 \mathrm{~min}$. Masson staining was used to dye the collagen fibers blue. Sirius red staining was performed at room temperature $\left(25^{\circ} \mathrm{C}\right)$ for $1 \mathrm{~h}$. Following Sirius red staining, the collagen fibers were stained red. For PASM staining, the sections were stained with periodic acid for $15 \mathrm{~min}$ at room temperature $\left(25^{\circ} \mathrm{C}\right)$ and hexamine silver working solution for $40 \mathrm{~min}$ at $58^{\circ} \mathrm{C}$. Following PASM staining, elastic and mesh fibers were stained in black. The aforementioned staining was observed using a light microscope (Nikon Eclipse E100) at a magnification of $x 400$. The sum of integral optical density (IOD) was used to quantify the results and calculated using Image-Pro Plus 6.0 software (Media Cybernetics, Inc.).

Immunohistochemistry assays. The 4- $\mu \mathrm{m}$-thick sections were baked at $60^{\circ} \mathrm{C}$ for $2 \mathrm{~h}$ and then deparaffinized in xylene, rehydrated in 100, 85 and $75 \%$ alcohol, and then washed in water. Subsequently, antigen retrieval was performed. The samples were treated with $3 \%$ hydrogen peroxide to block endogenous peroxidase activity and then blocked with BSA for $30 \mathrm{~min}$ at room temperature $\left(25^{\circ} \mathrm{C}\right)$. Sections were incubated with vimentin antibody (1:500; Wuhan Servicebio Technology
Co., Ltd.; cat. no. GB11192) at $4^{\circ} \mathrm{C}$ overnight. Subsequently, the appropriate secondary antibodies (1:200; HRP-goat anti-rabbit IgG; Wuhan Servicebio Technology Co., Ltd.; cat. no. GB23303) were incubated with the sections in the dark at room temperature $\left(25^{\circ} \mathrm{C}\right)$ for $50 \mathrm{~min}$. Subsequently, 3,3'-diaminobenzidine dye solution (Dako; Agilent Technologies, Inc.) was incubated with the sections at room temperature in the dark. Sections were observed and images were acquired using a light microscope (magnification, $x 400$ ). The sum of IOD was used to quantify the results and calculated using Image-Pro Plus 6.0 software (Media Cybernetics, Inc.). The color of vimentin staining was claybank.

Immunofluorescence method. The 4- $\mu$ m-thick sections were baked at $60^{\circ} \mathrm{C}$ for $2 \mathrm{~h}$ and then deparaffinized in xylene, rehydrated in 100, 85 and $75 \%$ alcohol, and then washed in water. Subsequently, antigen retrieval was performed. Then, to reduce the spontaneous auto-fluorescence, tissue auto-fluorescence quencher (Wuhan Servicebio Technology Co., Ltd.; cat. no. G1221) was added for $5 \mathrm{~min}$ and samples were rinsed in water for $10 \mathrm{~min}$. Sections were blocked using BSA for $30 \mathrm{~min}$ at room temperature $\left(25^{\circ} \mathrm{C}\right)$. Samples were incubated with anti-E-cadherin (1:5,000; Wuhan Servicebio Technology Co., Ltd.; cat. no. GB12082) and anti- $\alpha$-smooth muscle actin ( $\alpha$-SMA) antibodies (1:10,000; Wuhan Servicebio Technology Co., Ltd.; cat. no. GB13044) at $4^{\circ} \mathrm{C}$ overnight. Following incubation with the primary antibodies, the sections were washed and incubated with the corresponding secondary antibodies (1:500; HRP-goat anti-mouse IgG; Wuhan Servicebio Technology Co., Ltd.; cat. no. GB23301) in the dark at room temperature $\left(25^{\circ} \mathrm{C}\right)$ for 50 min. DAPI solution (Wuhan Servicebio Technology Co., Ltd.) was then added and the samples were incubated in the dark at room temperature for $10 \mathrm{~min}$. An anti-fluorescence quenching solution was used to seal the samples. Samples were observed and images were acquired using a confocal microscope (Nikon Corporation; magnification, x400). The sum of IOD was used to quantify the results and calculated using Image-Pro Plus 6.0 software (Media Cybernetics, Inc.). E-cadherin was stained in red and $\alpha$-SMA was stained in green.

RNA library construction and sequencing. Suzhou Basepair Biotechnology Company (http://www.basepair.cn/) performed the RNA library construction and sequencing in biological triplicates using an Illumina HiSeq X Ten Sequencing system (Illumina, Inc.). The main sequencing processes included sequencing data quality preprocessing, reference genome alignment, gene expression analysis and differential expression analysis $(25,27)$.

Kyoto Encyclopedia of Genes and Genomes (KEGG) pathway enrichment. Sequencing data were analyzed as raw reads, and were saved in a FASTQ format document. To obtain clean reads, adaptor-contaminated and low-quality sequences were removed using filtering methods, as previously described (28). FastQC (version 0.11.4; http://www.bioinformatics.babraham. ac.uk/projects/fastqc/) was used to examine the quality of the clean reads (25). Subsequently, the reads were aligned to the reference genome Ensemble-Rnor6.0 using Hisat2 
A

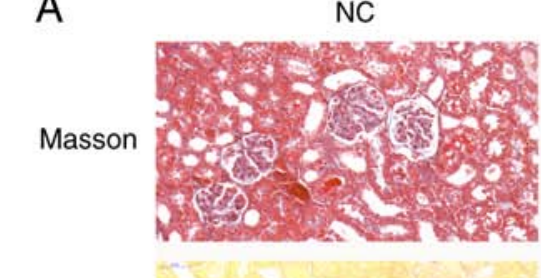

Sirius red
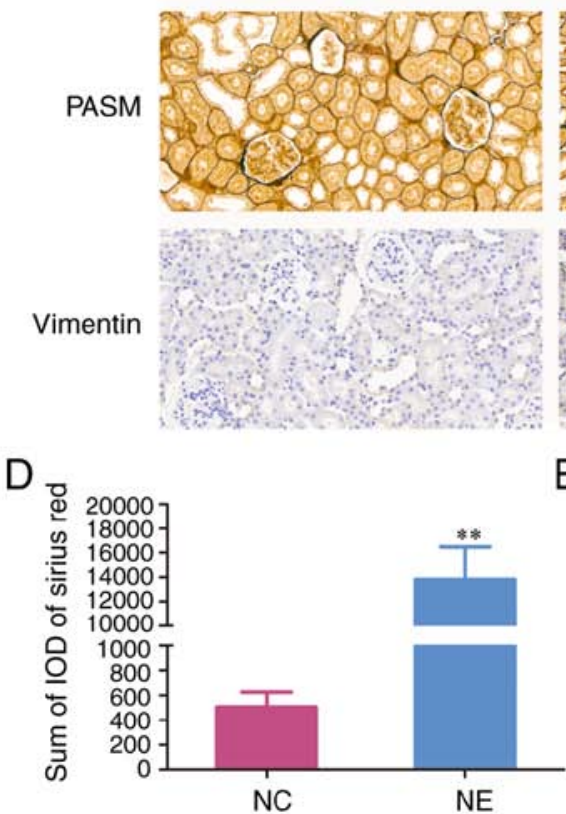
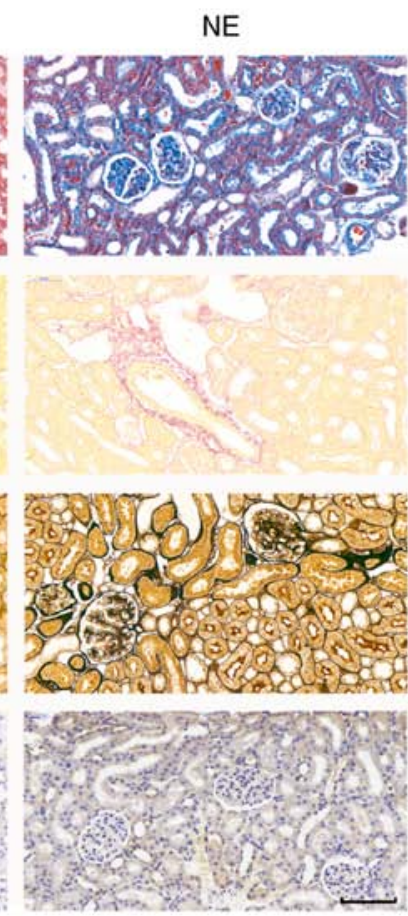

E

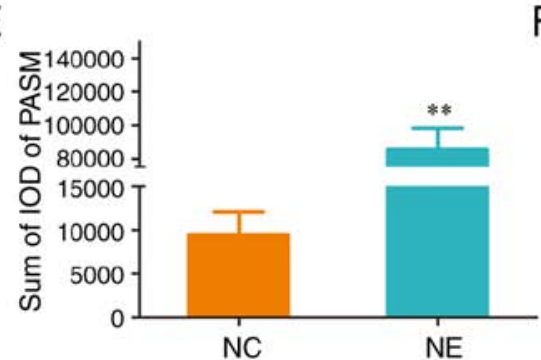

B

C
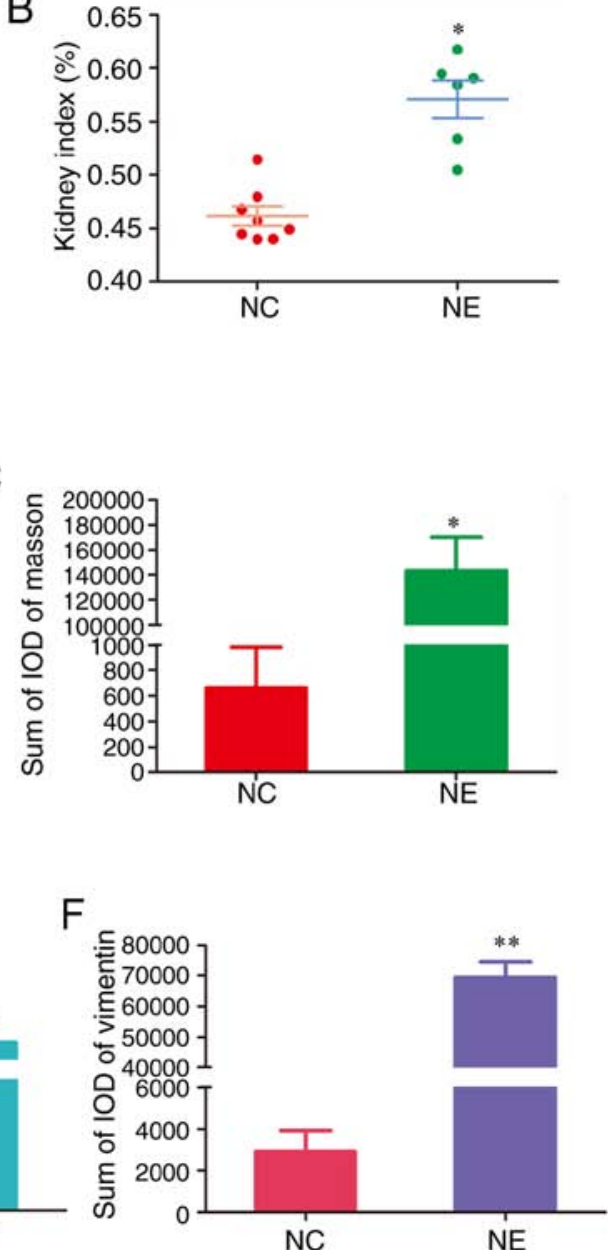

Figure 1. Renal histology analysis. (A) Pathological staining. Scale bar, $100 \mu \mathrm{m}$. Magnification, $\mathrm{x} 400$. (B) Kidney index, kidney index $=$ average kidney weight/body weight. (C) Sum of IOD of Masson staining. (D) Sum of IOD of Sirius red staining. (E) Sum of IOD of PASM staining. (F) Sum of IOD of Vimentin expression. ${ }^{*} \mathrm{P}<0.05,{ }^{* *} \mathrm{P}<0.01$ vs. NC. NC, normal control group; NE, nephrotoxicity group; IOD, integral optical density; PASM, periodic acid-silver metheramine.

(version 2.1.0). Gene coverage was analyzed using the percentage of genes covered by the reads. Functional annotation was performed using ANNOVAR (29). Differentially expressed genes (DEGs) were identified using the DEGseq panormalage method (30), according to negative binomial distributions. The gene expression levels were analyzed according to the number of fragments per kilobase of transcript per million reads and counts values. Genes with an adjusted $\log _{2}$ (fold change) $\mid>1$ and $\mathrm{P}<0.05$ were considered as DEGs. KEGG pathway analysis was performed for the DEGs using the KEGG Orthology-Based Annotation System (31). The significantly enriched KEGG pathways exhibited a $\mathrm{P}<0.05(25,27,28,32)$.

Statistical analysis. Data are presented as the mean \pm standard error of the mean. The statistical analysis was performed using SPSS (version 13.0; SPSS, Inc. and GraphPad Prism (version 5.0; GraphPad Software Inc.). Each experiment was performed three times. An unpaired Student's t-test was used to compare the protein expression level between the two groups. $\mathrm{P}<0.05$ was considered to indicate a statistically significant difference.

\section{Results}

Pathological changes in kidneys induced by tacrolimus. Kidneys were examined using pathological and immunofluorescent staining (Figs. 1A and 2A, respectively). The NE group exhibited a significantly higher of kidney index compared with the NC group (Fig. 1B; P $<0.05$ ). Compared with the NC group, the NE group exhibited severe renal fibrosis. This was indicated by a significantly higher sum of IOD in the NE group following Masson staining (Fig. 1C; $\mathrm{P}<0.05$ ), Sirius red staining (Fig. 1D; P<0.01) and PASM staining (Fig. 1E; $\mathrm{P}<0.01)$. In addition, the NE group exhibited significantly upregulated vimentin (Fig. 1F; $\mathrm{P}<0.01$ ), significantly downregulated $\mathrm{E}$-cadherin (Fig. $2 \mathrm{~B} ; \mathrm{P}<0.05)$ and significantly upregulated $\alpha$-SMA (Fig. 2C; P<0.01).

Transcriptomic and bioinformatics analyses reveal the nephrotoxicity mechanism underlying tacrolimus. A total of six next-generation sequencing libraries, including three from the NC group and three from the NE group, were analyzed. As presented in Fig. 3, the transcriptomic analysis identified 453 DEGs, including 173 upregulated and 280 downregulated 

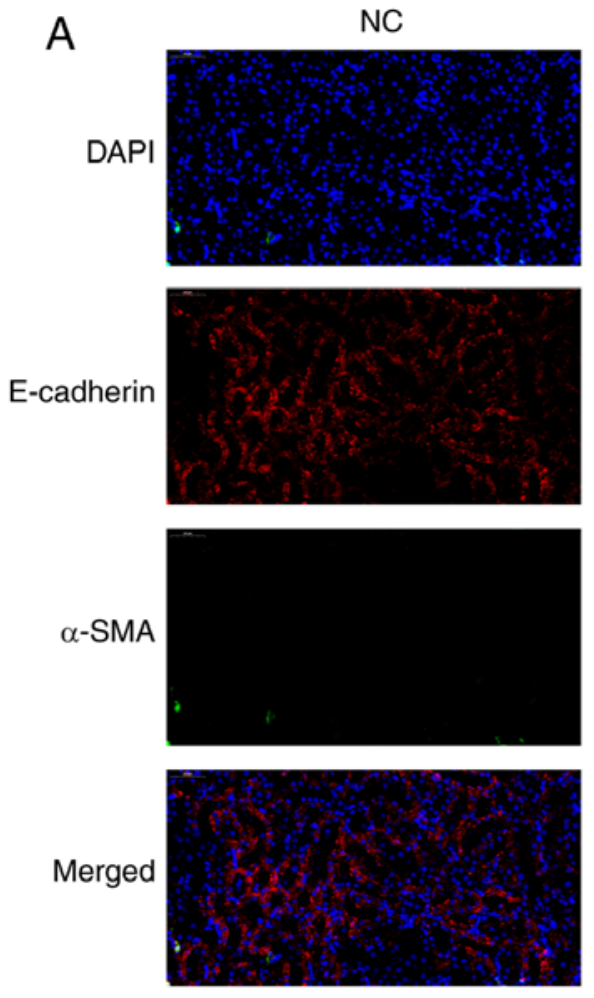
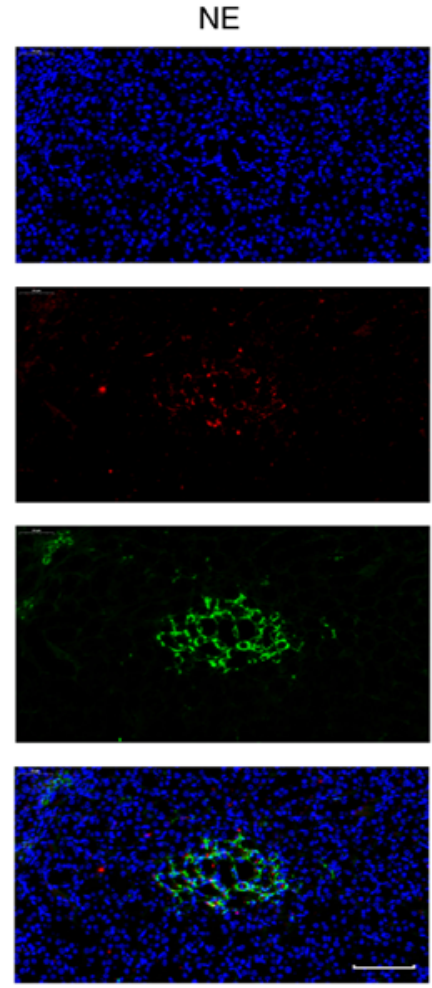
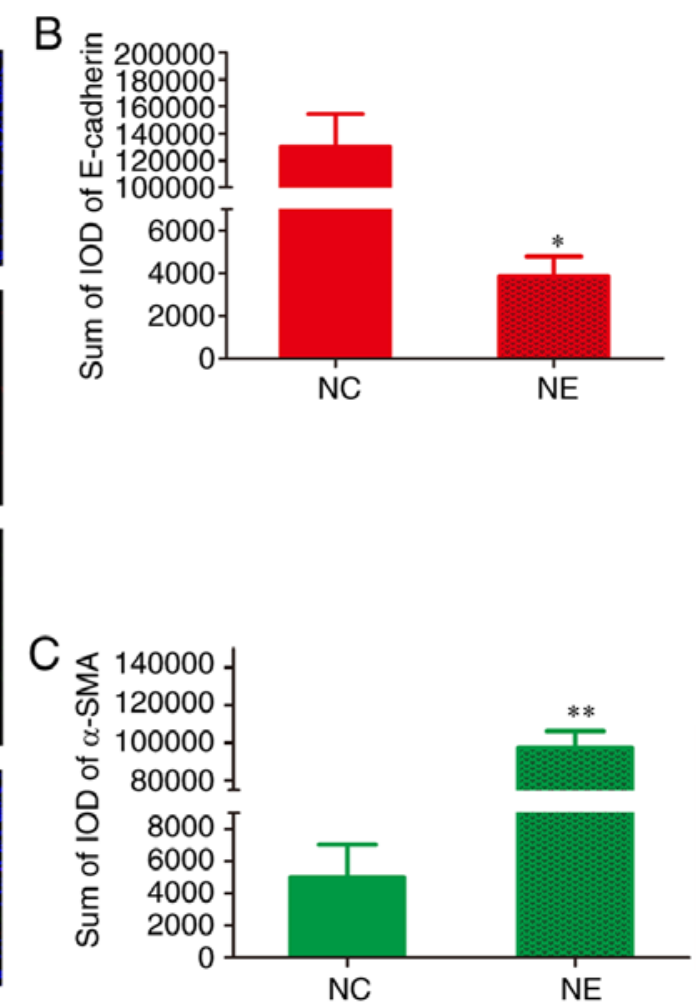

Figure 2. Expression of E-cadherin and $\alpha$-SMA. (A) Immunofluorescence staining. Scale bar, $100 \mu \mathrm{m}$, Magnification, $\mathrm{x} 400$. (B) Sum of IOD of E-cadherin expression. (C) Sum of IOD of $\alpha$-SMA expression. ${ }^{*} \mathrm{P}<0.05,{ }^{* *} \mathrm{P}<0.01$ vs. NC. NC, normal control group; NE, nephrotoxicity group; $\alpha$-SMA, $\alpha$-smooth muscle actin; IOD, integral optical density.

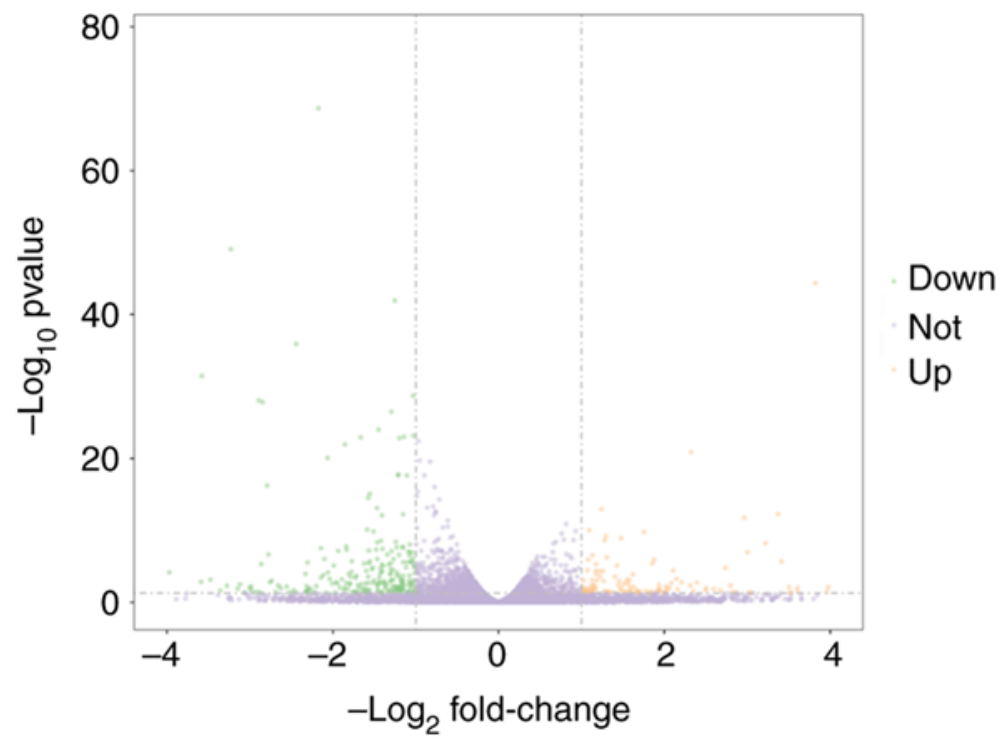

Figure 3. Differentially expressed genes of the NE and NC groups identified by $\operatorname{llog} 2$ (fold change) $>1$ and $\mathrm{P}<0.05$. NC, normal control group. NE, nephrotoxicity group.

genes. The pathway demonstrating the highest enrichment following KEGG analysis was 'cytokine-cytokine receptor interaction' (Fig. 4A; $\mathrm{P}<0.05$ ). Further analysis of this signaling pathway using KEGG identified that tacrolimus increased the expression levels of CXCL1, CXCL2, CXCL3 and the chemokine receptor CXCR2 (Fig. 4B; $\mathrm{P}<0.05$ ). The potential mechanism underlying tacrolimus-induced nephrotoxicity is presented in Fig. 5.

\section{Discussion}

Tacrolimus is one of the most used and effective clinical immunosuppressive agents currently available in the clinic, and it has been widely used for treating patients receiving renal (33-38), liver (39-44) and lung transplants (45), and for patients with idiopathic membranous nephropathy (46), nephritic syndrome (4) and systemic-onset juvenile idio- 
A

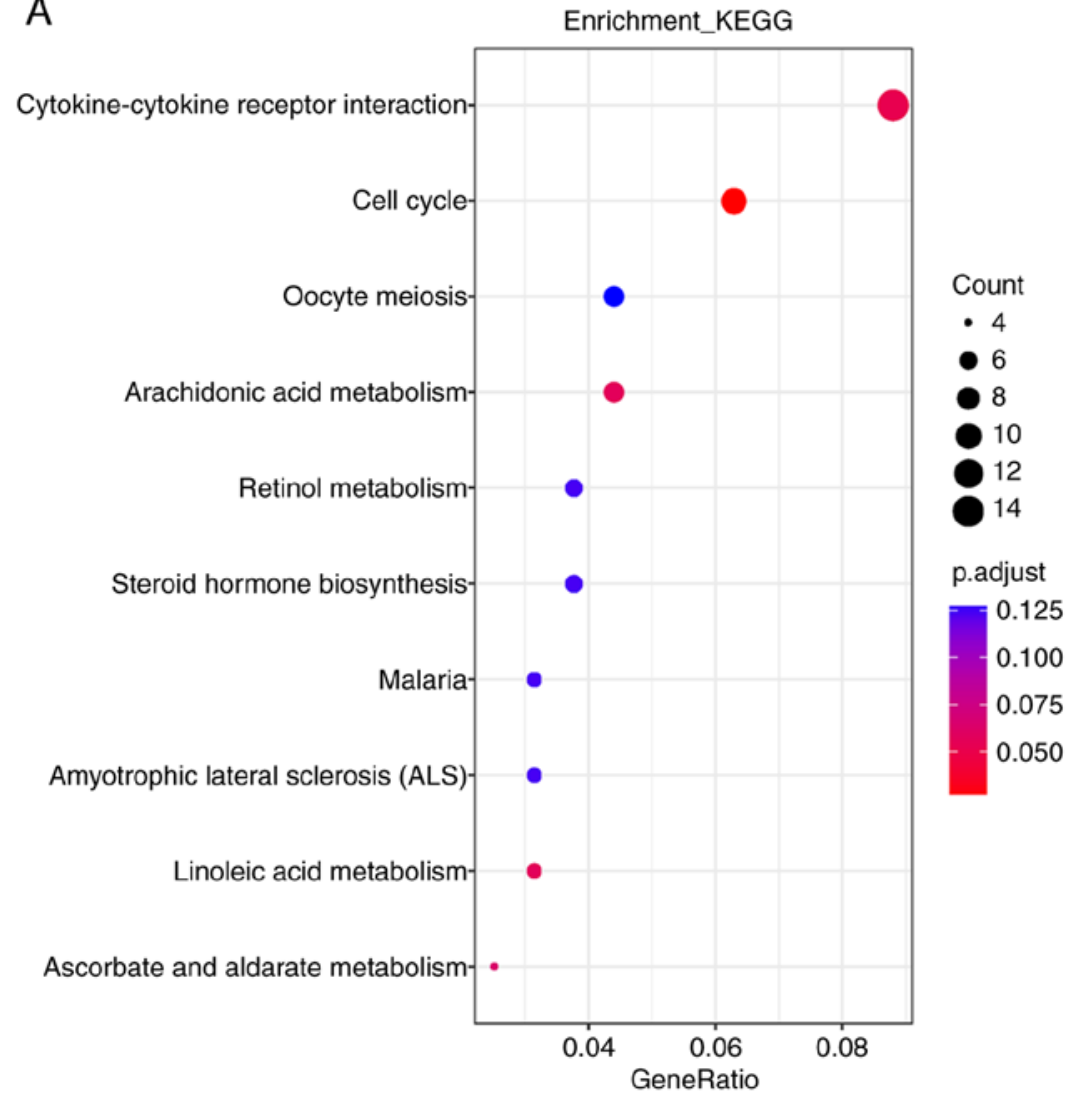

B

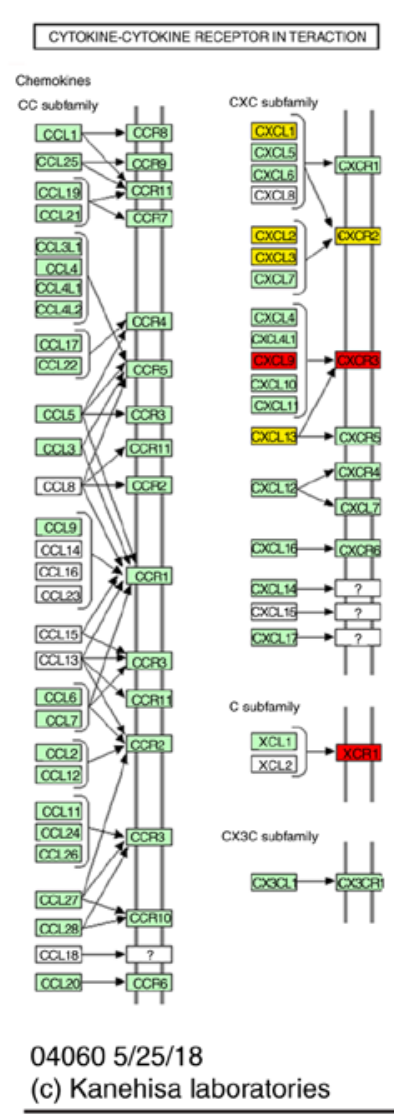

Figure 4. Differential gene expression screening. (A) KEGG pathway enrichment of differentially expressed genes. (B) The pathway map of cytokine-cytokine receptor interaction. Yellow indicates upregulated genes, red indicates downregulated genes and green indicates that the gene is both upregulated and downregulated. KEGG, Kyoto Encyclopedia of Genes and Genomes.

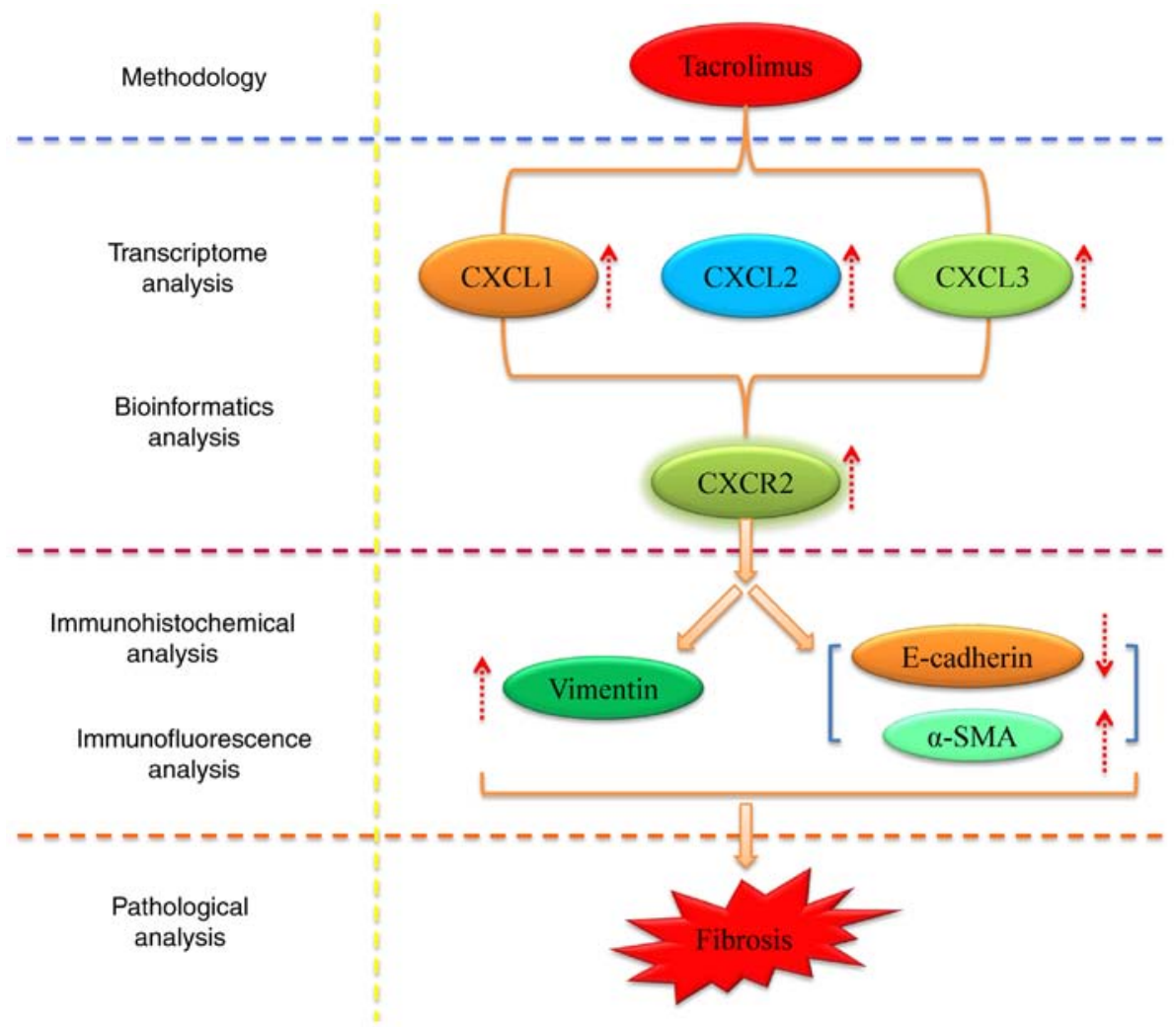

Figure 5. Potential mechanism of nephrotoxicity induced by tacrolimus. Tacrolimus increases the expression levels of CXCL1, CXCL2 and CXCL3 and the chemokine receptor CXCR2, which further promotes renal fibrosis progression. CXCL, chemokine (C-X-C) motif ligand; CXCR2, C-X-C chemokine receptor type $2 ; \alpha$-SMA, $\alpha$-smooth muscle actin. 
pathic arthritis (7). However, although tacrolimus has been demonstrated to exhibit evident benefits, immunosuppressive agents are associated with the occurrence of acute or chronic renal toxicity, limiting their clinical use (15).

In addition, it has previously been reported that the epithet-lial-mesenchymal transition (EMT) is significantly correlated with renal fibrosis, which is associated with calcineurin inhibitor-mediated nephrotoxicity (21). EMT is one of the basic mechanisms of renal fibrosis and involves various processes in which epithelial cells stop exhibiting epithelial characteristics, including the expression of E-cadherin, and obtain traits specific of mesenchymal cells, including the upregulation of $\alpha$-SMA $(25,47-48)$. In addition, it has previously been reported that vimentin is a potential novel biomarker in renal fibrosis $(25,49)$.

In the present study, Masson staining, Sirius red staining and PASM staining were used to examine the pathological alterations occurring in kidneys. Compared with the NC group, the tacrolimus nephrotoxicity group exhibited severe renal fibrosis. Further analysis confirmed that vimentin was upregulated, E-cadherin was downregulated and $\alpha$-SMA was upregulated in the tacrolimus-induced nephrotoxicity group. Subsequently, the exact mechanism underlying tacrolimus-induced nephrotoxicity was examined.

Transcriptome analysis can identify both coding and non-coding RNA, quantifying gene expression heterogeneity in cells, tissues, organs and even in the whole organism (50). Transcriptome analysis is important to investigate various processes (51) and it has been widely used to identify key factors in the progression of multiple diseases (27,52-53). Kim et al (52), using transcriptome analysis, identified Tensin 4 as a key effector of cetuximab and a regulator of the oncogenic activity of KRAS-mutant colorectal cancer cell lines. Yang et al (27) reported the transcriptome profiling of brain myeloid cells, and identified an upregulation of integrin subunit $\alpha \mathrm{L}$, triggering receptor expressed on myeloid cells 1 and secreted phosphoprotein 1 in Western diet-induced obesity. Siena et al (53) performed a whole transcriptome analysis in melanoma and identified a correlation between the expression level of the long non-coding RNA ZEB1-AS1 with invasive ability of melanoma cells. These previous studies suggested that the transcriptome has become a reliable tool to identify key factors in the development and progression of various diseases $(27,52,53)$. The present study aimed to investigate the mechanism underlying tacrolimus nephrotoxicity and to identify novel potential targets via transcriptomic and bioinformatics analyses.

The KEGG enrichment analysis identified 'cytokine-cytokine receptor interaction' as the pathway most significantly enriched following tacrolimus-mediated nephrotoxicity induction. By analyzing components of the "cytokine-cytokine receptor interaction' signaling pathway, tacrolimus was identified to increase the expression levels of CXCL1, CXCL2, CXCL3 and the chemokine receptor CXCR2.

CXCR2 is a seven-transmembrane G-protein-coupled receptor that mediates chemotaxis during immune response, and is expressed in renal parenchymal cells and neutrophils (54-55). Dornelles et al (56) firstly reported the association between the increase in CXCR2 expression and nephrotoxicity following cyclophosphamide treatment.
In addition, upregulation of CXCR2 has been reported in inflammatory diseases, including psoriasis, atherosclerosis and rheumatoid arthritis $(57,58)$. CXCR2-knockout mice were identified to be protected against dextran sodium sulfate-mediated colitis and acute kidney injury. In addition, the expression of cytokines and chemokines and the level of neutrophil infiltration were reduced in the colon and kidney of CXCR2-knockout mice (59). Collectively, these previous studies suggest that CXCR2 may be a promoter of kidney damage.

In conclusion, the mechanism underlying tacrolimus-induced nephrotoxicity may involve the increase of the chemokine receptor CXCR2 to promote the upregulation of vimentin and $\alpha$-SMA, and the downregulation of E-cadherin, thus accelerating the renal fibrosis progression. However, the present analysis was performed in animal models, and validation of the present results is required in the future by analyzing blood and kidney biopsies from patients with nephrotoxicity caused by tacrolimus.

\section{Acknowledgements}

Not applicable.

\section{Funding}

This study was supported by Clinical Pharmacy Key Specialty Construction Project of Shanghai (grant no. YZ2017/5), Important Weak Subject Construction Project of Shanghai (grant no. 2016ZB0305), Scientific Research Project of Science and Technology Commission of Shanghai Municipality (grant no. 18DZ1910604) and the China Scholarship Council (grant no. 201906100164).

\section{Availability of data and materials}

The datasets used and/or analyzed during the current study are available from the corresponding author on reasonable request.

\section{Authors' contributions}

ZL and HX conceived and designed the study. DW, XC and MF performed the experiments. DW and XC wrote, reviewed and edited the manuscript. All authors read and approved the final manuscript.

\section{Ethics approval and consent to participate}

Animal protocols and procedures were approved by The Animal Care and Use Committee of Children's Hospital of Fudan University (Shanghai, China) and complied with the appropriate institutional regulations.

\section{Patient consent for publication}

Not applicable.

\section{Competing interests}

The authors declare that they have no competing interests. 


\section{References}

1. Yoshida T, Nakanishi K, Yoshioka T, Tsutsui Y, Maeda A, Kondo $\mathrm{H}$ and Sako K: Oral tacrolimus oil formulations for enhanced lymphatic delivery and efficient inhibition of T-cell's interleukin-2 production. Eur J Pharm Biopharm 100: 58-65, 2016.

2. Maguire O, Tornatore KM, O'Loughlin KL, Venuto RC and Minderman H: Nuclear translocation of nuclear factor of activated $\mathrm{T}$ cells (NFAT) as a quantitative pharmacodynamic parameter for tacrolimus. Cytometry A 83: 1096-1104, 2013.

3. Dong QE, Fu R, Liu CY, Ruan EB, Wang XM, Wang GJ, Qu W, Liu $\mathrm{H}$, Wu YH, Song J, et al: Inhibitory effects of tacrolimus on effector $\mathrm{T}$ cells from patients with severe aplastic anemia. Zhonghua Yi Xue Za Zhi 93: 1541-1545, 2013 (In Chinese).

4. Wang DD, Chen X and Li ZP: Efficacy and safety of tacrolimus in treating pediatric refractory nephrotic syndrome: A meta-analysis. Int J Clin Exp Med 11: 6436-6444, 2018.

5. Ho S, Clipstone N, Timmermann L, Northrop J, Graef I, Fiorentino D, Nourse J and Crabtree GR: The mechanism of action of cyclosporin A and FK506. Clin Immunol Immunopathol 80: S40-S45, 1996.

6. Masuda S and Inui K: An up-date review on individualized dosage adjustment of calcineurin inhibitors in organ transplant patients. Pharmacol Ther 112: 184-198, 2006.

7. Wang D, Chen $X$ and Li Z: Treatment of patients with systemic-onset juvenile idiopathic arthritis with tacrolimus. Exp Ther Med 17: 2305-2309, 2019.

8. Wang D, Chen X, Xu H and Li Z: Population pharmacokinetics and dosing regimen optimisation of tacrolimus in Chinese pediatric hematopoietic stem cell transplantation patients. Xenobiotica 2: 1-8, Apr 2, 2019 (Epub ahead of print).

9. Wang DD, Chen X and Li ZP: Wuzhi capsule and haemoglobin influence tacrolimus elimination in paediatric kidney transplantation patients in a population pharmacokinetics analysis: A retrospective study. J Clin Pharm Ther 44: 611-617, 2019.

10. Wang DD, Chen X and Li ZP: Population pharmacokinetics of sirolimus in pediatric patients with kaposiform hemangioendothelioma: A retrospective study. Oncol Lett 18: 2412-2419, 2019.

11. Wang DD, Chen X and Li ZP: Efficacy and safety of tacrolimus in Chinese lupus nephritis patients: A meta-analysis. Int J Clin Exp Med 12: 3056-3064, 2019.

12. Jusko WJ, Thomson AW, Fung J, McMaster P, Wong SH, Zylber-Katz E, Christians U, Winkler M, Fitzsimmons WE and Lieberman R: Consensus document: Therapeutic monitoring of tacrolimus (FK-506). Ther Drug Monit 17: 606-614, 1995.

13. Venkataramanan R, Swaminathan A, Prasad T, Jain A, Zuckerman S, Warty V, McMichael J, Lever J, Burckart G and Starzl T: Clinical pharmacokinetics of tacrolimus. Clin Pharmacokinet 29: 404-430, 1995.

14. Schutte-Nutgen K, Tholking G, Suwelack B and Reuter S: Tacrolimus-pharmacokinetic considerations for clinicians. Curr Drug Metab 19: 342-350, 2018.

15. Staatz CE and Tett SE: Clinical pharmacokinetics and pharmacodynamics of tacrolimus in solid organ transplantation. Clin Pharmacokinet 43: 623-653, 2004.

16. Joardar S, Dewanjee S, Bhowmick S, Dua TK, Das S, Saha A and De Feo V: Rosmarinic acid attenuates cadmium-induced nephrotoxicity via inhibition of oxidative stress, apoptosis, inflammation and fibrosis. Int J Mol Sci 20: E2027, 2019.

17. Gonzalez-Guerrero C, Cannata-Ortiz P, Guerri C, Egido J, Ortiz A and Ramos AM: TLR4-mediated inflammation is a key pathogenic event leading to kidney damage and fibrosis in cyclosporine nephrotoxicity. Arch Toxicol 91: 1925-1939, 2017.

18. Al-Gayyar MM, Hassan HM, Alyoussef A, Abbas A, Darweish MM and El-Hawwary AA: Nigella sativa oil attenuates chronic nephrotoxicity induced by oral sodium nitrite: Effects on tissue fibrosis and apoptosis. Redox Rep 21: 50-60, 2016.

19. Nielsen FT, Jensen BL, Hansen PB, Marcussen N and Bie P: The mineralocorticoid receptor antagonist eplerenone reduces renal interstitial fibrosis after long-term cyclosporine treatment in rat: Antagonizing cyclosporine nephrotoxicity. BMC Nephrol 14: 42, 2013.

20. Okada H, Watanabe Y, Inoue T, Kobayashi T, Kanno Y, Shiota G, Nakamura T, Sugaya T, Fukamizu A and Suzuki H: Transgene-derived hepatocyte growth factor attenuates reactive renal fibrosis in aristolochic acid nephrotoxicity. Nephrol Dial Transplant 18: 2515-2523, 2003.
21. Bennett J, Cassidy H, Slattery C, Ryan MP and McMorrow T: Tacrolimus modulates TGF- $\beta$ signaling to induce epithelial-mesenchymal transition in human renal proximal tubule epithelial cells. J Clin Med 5: E50, 2016.

22. Shihab FS, Bennett WM, Tanner AM and Andoh TF: Mechanism of fibrosis in experimental tacrolimus nephrotoxicity. Transplantation 64: 1829-1837, 1997.

23. Mitamura T, Yamada A, Ishida H, Fujihira S, Ohara K, Noguchi $H$ and Mine Y: Tacrolimus (FK506)-induced nephrotoxicity in spontaneous hypertensive rats. J Toxicol Sci 19: 219-226, 1994.

24. Al-Harbi NO, Imam F, Al-Harbi MM, Iqbal M, Nadeem A, Al-Shahrah OA, Korashy HM, Al-Hosaini KA, Ahmed M and Bahashwar S: Treatment with aliskiren ameliorates tacrolimus-induced nephrotoxicity in rats. J Renin Angiotensin Aldosterone Syst 16: 1329-1336, 2015.

25. Wang D, Chen X, Fu M and Li Z: Transcriptomics analysis of sirolimus treatment in lupus nephritis. Mol Med Rep 20: 245-251, 2019.

26. Wang D, Zhang G, Chen X, Wei T, Liu C, Chen C, Gong Y and Wei Q: Sitagliptin ameliorates diabetic nephropathy by blocking TGF- $\beta 1 /$ Smad signaling pathway. Int J Mol Med 41: 2784-2792, 2018.

27. Yang H, Graham LC, Reagan AM, Grabowska WA, Schott WH and Howell GR: Transcriptome profiling of brain myeloid cells revealed activation of Itgal, Trem1, and Spp1 in western diet-induced obesity. J Neuroinflammation 16: 169, 2019.

28. Liu J, Yang L, Hou Y, Soteyome T, Zeng B, Su J, Li L, Li B, Chen D, Li Y, et al: Transcriptomics study on staphylococcus aureus biofilm under low concentration of ampicillin. Front Microbiol 9: 2413, 2018

29. Wang K, Li M and Hakonarson H: ANNOVAR: Functional annotation of genetic variants from high-throughput sequencing data. Nucleic Acids Res 38: e164, 2010.

30. Burden CJ, Qureshi SE and Wilson SR: Error estimates for the analysis of differential expression from RNA-seq count data. PeerJ 2: e576, 2014.

31. Kanehisa M and Goto S: KEGG: Kyoto encyclopedia of genes and genomes. Nucleic Acids Res 28: 27-30, 2000.

32. Zhang Q, Sun S, Zhu C, Zheng Y, Cai Q, Liang X, Xie H and Zhou J: Prediction and analysis of weighted genes in hepatocellular carcinoma using bioinformatics analysis. Mol Med Rep 19: 2479-2488, 2019.

33. Andreu F, Colom H, Grinyo JM, Torras J, Cruzado JM and Lloberas N: Development of a population PK model of tacrolimus for adaptive dosage control in stable kidney transplant patients. Ther Drug Monit 37: 246-255, 2015.

34. Benkali K, Rostaing L, Premaud A, Woillard JB, Saint-Marcoux F, Urien S, Kamar N, Marquet P and Rousseau A: Population pharmacokinetics and bayesian estimation of tacrolimus exposure in renal transplant recipients on a new once-daily formulation. Clin Pharmacokinet 49: 683-692, 2010.

35. Bergmann TK, Hennig S, Barraclough KA, Isbel NM and Staatz CE: Population pharmacokinetics of tacrolimus in adult kidney transplant patients: Impact of CYP3A5 genotype on starting dose. Ther Drug Monit 36: 62-70, 2014.

36. Han N, Ha S, Yun HY, Kim MG, Min SI, Ha J, Lee JI, Oh JM and Kim IW: Population pharmacokinetic-pharmacogenetic model of tacrolimus in the early period after kidney transplantation. Basic Clin Pharmacol Toxicol 114: 400-406, 2014.

37. Zhao W, Elie V, Roussey G, Brochard K, Niaudet P, Leroy V, Loirat C, Cochat P, Cloarec S, Andre JL, et al: Population pharmacokinetics and pharmacogenetics of tacrolimus in de novo pediatric kidney transplant recipients. Clin Pharmacol Ther 86: 609-618, 2009.

38. Zuo XC, Ng CM, Barrett JS, Luo AJ, Zhang BK, Deng CH, Xi LY, Cheng K, Ming YZ, Yang GP, et al: Effects of CYP3A4 and CYP3A5 polymorphisms on tacrolimus pharmacokinetics in Chinese adult renal transplant recipients: A population pharmacokinetic analysis. Pharmacogenet Genomics 23: 251-261, 2013.

39. Lu YX, Su QH, Wu KH, Ren YP, Li L, Zhou TY and Lu W: A population pharmacokinetic study of tacrolimus in healthy Chinese volunteers and liver transplant patients. Acta Pharmacol Sin 36: 281-288, 2015.

40. Musuamba FT, Guy-Viterbo V, Reding R, Verbeeck RK and Wallemacq P: Population pharmacokinetic analysis of tacrolimus early after pediatric liver transplantation. Ther Drug Monit 36: 54-61, 2014.

41. Wallin JE, Bergstrand M, Wilczek HE, Nydert PS, Karlsson MO and Staatz CE: Population pharmacokinetics of tacrolimus in pediatric liver transplantation: Early posttransplantation clearance. Ther Drug Monit 33: 663-672, 2011. 
42. Yang JW, Liao SS, Zhu LQ, Zhao Y, Zhang Y, Sun XY, Rao W, $\mathrm{Qu}$ W, Li WZ and Sun LY: Population pharmacokinetic analysis of tacrolimus early after Chinese pediatric liver transplantation. Int J Clin Pharmacol Ther 53: 75-83, 2015.

43. Zhang XQ, Wang ZW, Fan JW, Li YP, Jiao Z, Gao JW, Peng ZH and Liu GL: The impact of sulfonylureas on tacrolimus apparent clearance revealed by a population pharmacokinetics analysis in Chinese adult liver-transplant patients. Ther Drug Monit 34: 126-133, 2012

44. Zhu L, Yang J, Zhang Y, Jing Y, Zhang Y and Li G: Effects of CYP3A5 genotypes, ABCB1 C3435T and G2677T/A polymorphism on pharmacokinetics of Tacrolimus in Chinese adult liver transplant patients. Xenobiotica 45: 840-846, 2015.

45. Monchaud C, de Winter BC, Knoop C, Estenne M, Reynaud-Gaubert M, Pison C, Stern M, Kessler R, Guillemain R, Marquet $\mathrm{P}$ and Rousseau A: Population pharmacokinetic modelling and design of a bayesian estimator for therapeutic drug monitoring of tacrolimus in lung transplantation. Clin Pharmacokinet 51: 175-186, 2012.

46. Di J, Qian Q, Yang M, Jiang Y, Zhou H, Li M and Zou Y: Efficacy and safety of long-course tacrolimus treatment for idiopathic membranous nephropathy. Exp Ther Med 16: 979-984, 2018.

47. Chen X, Yang Y, Liu C, Chen Z and Wang D: Astragaloside IV ameliorates high glucoseinduced renal tubular epithelialmesenchymal transition by blocking mTORC1/p70S6K signaling in HK2 cells. Int J Mol Med 43: 709-716, 2019.

48. Badid C, Desmouliere A, Babici D, Hadj-Aissa A, McGregor B, Lefrancois N, Touraine JL and Laville M: Interstitial expression of alpha-SMA: An early marker of chronic renal allograft dysfunction. Nephrol Dial Transplant 17: 1993-1998, 2002.

49. Cao YH, Lv LL, Zhang X, Hu H, Ding LH, Yin D, Zhang YZ, Ni HF, Chen PS and Liu BC: Urinary vimentin mRNA as a potential novel biomarker of renal fibrosis. Am J Physiol Renal Physiol 309: F514-F522, 2015.

50. Granjeaud S, Bertucci F and Jordan BR: Expression profiling: DNA arrays in many guises. Bioessays 21: 781-790, 1999.

51. Jiang Z, Zhou X, Li R, Michal JJ, Zhang S, Dodson MV, Zhang $\mathrm{Z}$ and Harland RM: Whole transcriptome analysis with sequencing: Methods, challenges and potential solutions. Cell Mol Life Sci 72: 3425-3439, 2015.
52. Kim S, Kim N, Kang K, Kim W, Won J and Cho J: Whole transcriptome analysis identifies TNS4 as a key effector of cetuximab and a regulator of the oncogenic activity of KRAS mutant colorectal cancer cell lines. Cells 8: 878, 2019.

53. Siena ÁDD, Plaça JR, Araújo LF, de Barros II, Peronni K, Molfetta G, de Biagi CAO Jr, Espreafico EM, Sousa JF and Silva WA Jr: Whole transcriptome analysis reveals correlation of long noncoding RNA ZEB1-AS1 with invasive profile in melanoma. Sci Rep 9: 11350, 2019.

54. Svensson M, Irjala H, Svanborg C and Godaly G: Effects of epithelial and neutrophil CXCR2 on innate immunity and resistance to kidney infection. Kidney Int 74: 81-90, 2008.

55. Li L, Huang L, Vergis AL, Ye H, Bajwa A, Narayan V, Strieter RM, Rosin DL and Okusa MD: IL-17 produced by neutrophils regulates IFN-gamma-mediated neutrophil migration in mouse kidney ischemia-reperfusion injury. J Clin Invest 120: 331-342, 2010.

56. Dornelles FN, Andrade EL, Campos MM and Calixto JB: Role of CXCR2 and TRPV1 in functional, inflammatory and behavioural changes in the rat model of cyclophosphamide-induced haemorrhagic cystitis. Br J Pharmacol 171: 452-467, 2014.

57. Mihara K, Smit MJ, Krajnc-Franken M, Gossen J, Rooseboom M and Dokter W: Human CXCR2 (hCXCR2) takes over functionalities of its murine homolog in hCXCR 2 knockin mice. Eur J Immunol 35: 2573-2582, 2005.

58. Liu L, Sun H, Wu S, Tan H, Sun Y, Liu X, Si S, Xu L, Huang J, Zhou W, et al: IL17A promotes CXCR2dependent angiogenesis in a mouse model of liver cancer. Mol Med Rep 20: 1065-1074, 2019.

59. Ranganathan P, Jayakumar C, Manicassamy S and Ramesh G: CXCR2 knockout mice are protected against DSS-colitis-induced acute kidney injury and inflammation. Am J Physiol Renal Physiol 305: F1422-F1427, 2013.

(i) 9 This work is licensed under a Creative Commons Attribution-NonCommercial-NoDerivatives 4.0 International (CC BY-NC-ND 4.0) License. 